\title{
Qualidade de vida dos profissionais de enfermagem atuantes em setores críticos
}

\author{
Quality of life of nursing professionals acting in critical sectors
}

\section{Calidad de vida de los profesionales de enfermería que actúan en sectores críticos}

\author{
Verusca Soares de Souza ${ }^{1}$, Daniela Siqueira da Silva ${ }^{\mathbb{D}}$, Liziane Viana Lima ${ }^{\mathbb{D}}$, Elen Ferraz Teston ${ }^{4}$, \\ Gabriella Michel dos Santos Benedetti $\left.{ }^{(}\right)$, Maria Antônia Ramos Costa ${ }^{6}\left(\mathbb{B}^{2}\right.$, Renata Rodrigues Mendonça ${ }^{7}$
}

\section{Histórico}

Recibido:

02 de febrero de 2018

Aceptado:

18 de abril de 2018

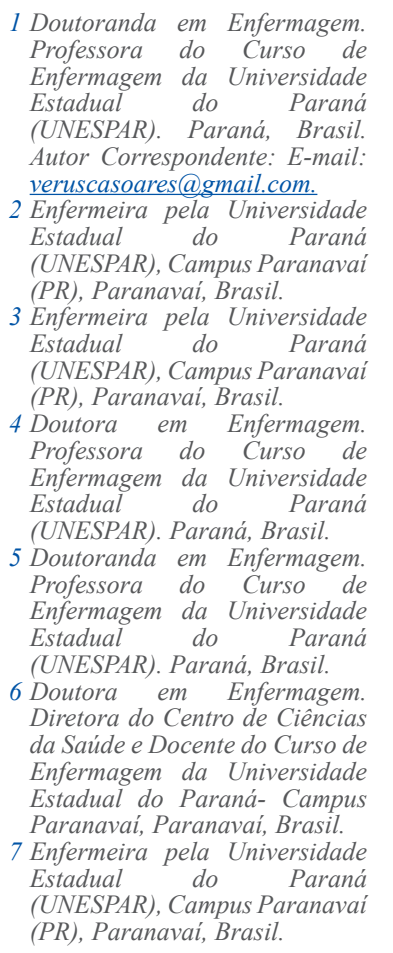

(PR), Paranavai, Brasil.
Introdução: Os serviços desenvolvidos em setores críticos são destinados a pacientes que necessitam de cuidados especializados em tempo hábil, tomada de decisão correta e utilização de recursos tecnológicos avançados para monitorização constante. $\mathrm{O}$ objetivo do estudo foi analisar a qualidade de vida de profissionais de enfermagem atuantes em setores críticos. Materiais e Métodos: Estudo transversal, quantitativo, com 75 profissionais de enfermagem de setores críticos. Destes, 11 do Pronto Socorro; 26 do Centro Cirúrgico; 23 da Unidade de Terapia Intensiva Adulto e; 15 da Unidade de Terapia Intensiva Neonatal de um hospital filantrópico, os quais responderam ao questionário auto aplicado sobre qualidade de vida (WHOQOL-BREF), que foi submetido à análise descritiva. Resultados: A maioria dos participantes era do sexo feminino (78,7\%), idade média de 30 anos, casado (53,3\%) e obtiveram escore total elevado de qualidade de vida $(73,33)$. O domínio Relacões Sociais foi o melhor avaliado e o que recebeu o pior escore foi o domínio Físico. Discussão: O domínio físico se relaciona ao sono/repouso, o exercício do trabalho em turnos ou plantões é um fator gerador de estresse, e pode influenciar na saúde e na qualidade de vida dos profissionais. Conclusões: Os profissionais de enfermagem possuem uma qualidade de vida satisfatória, entretanto, questões relacionadas à saúde física e a estrutura da instituição ainda se apresentam como fatores que interferem na qualidade de vida dos trabalhadores.

Palavras chave: Qualidade de Vida; Saúde do Trabalhador; Estresse Ocupacional; Cuidados Críticos.

Abstract

Introduction: Services offered in critical sectors are intended for patients needing timely skilled care, making the right decisions, and using advanced technological resources for constant monitoring. The aim of this study was to analyze the quality of life of nursing professionals working in critical sectors. Materials and Methods: Crosssectional, quantitative study with 75 nursing professionals from critical sectors. Of these, 11 from the Emergency Room; 26 from the Surgical Center; 23 from the Adult Intensive Care Unit; 15 from the Neonatal Intensive Care Unit in a philanthropic hospital, who answered the self-administered quality-of-life questionnaire (WHOQOL$B R I E F)$, which was subjected to descriptive analysis. Results: Most of the participants were female gender $(78.7 \%)$, with a mean age of 30 years, married $(53.3 \%)$ and obtained a high total quality-of-life score $(73.33)$. The Social Relations domain was the best evaluated and the one receiving the worst score was the Physical domain. Discussion: The physical domain is related to sleep/rest; work of shift or shifts is a factor that generates stress and can influence the health and quality of life of professionals. Conclusions: Nursing professionals have a satisfactory quality of life; however, issues related to physical health and the institution's structure are still factors that interfere in the quality of life of workers.

Key words: Quality of Life; Occupational Health; Burnout, Professional; Critical Care.

Resumen

Introducción: Los servicios desarrollados en sectores críticos son destinados a pacientes que necesitan cuidados especializados en tiempo oportuno, toma de decisión correcta y utilización de recursos tecnológicos avanzados para monitorización constante. El objetivo del estudio fue analizar la calidad de vida de los profesionales de enfermería que actúan en sectores críticos. Materiales y Métodos: Estudio transversal, cuantitativo, con 75 profesionales de enfermería de sectores críticos. De ellos, 11 del Pronto Socorro, 26 del Centro Quirúrgico, 23 de la Unidad de Terapia Intensiva Adulto y 15 de la Unidad de Terapia Intensiva Neonatal de un hospital filantrópico, los cuales respondieron al cuestionario auto-diligenciado sobre calidad de vida (WHOQOL-BREF), que fue sometido a análisis descriptivo. Resultados: La mayoría de los participantes eran del sexo femenino (78.7\%), edad media de 30 años, casado (53.3\%) y obtuvieron una puntuación total elevada de calidad de vida (73.33). El dominio Relaciones Sociales fue el mejor evaluado y el que recibió el peor puntaje fue el dominio Físico. Discusión: El dominio físico se relaciona con el sueño/reposo, el ejercicio del trabajo en turnos o plantones es un factor generador de estrés y puede influir en la salud y la calidad de vida de los profesionales. Conclusiones: Los profesionales de enfermería poseen una calidad de vida satisfactoria, sin embargo, cuestiones relacionadas a la salud física y la estructura de la institución todavía se presentan como factores que interfieren en la calidad de vida de los trabajadores.

Palabras clave: Calidad de Vida; Salud Laboral; Estrés Laboral; Cuidados Críticos.

Como citar este artigo: Souza VS, Silva DS, Lima LV, Teston EF, Benedetti GMS, Costa MAR, et al. Qualidade de vida dos profissionais de enfermagem atuantes em setores críticos. Rev Cuid. 2018; 9(2): 2177-86. http://dx.doi.org/10.15649/cuidarte.v9i2.506

(1) (1) (C)2018 Universidad de Santander. Este es un artículo de acceso abierto, distribuido bajo los términos de la licencia Creative Commons Attribution (CC BY-NC 4.0), que permite el uso ilimitado, distribución y reproducción en cualquier medio, siempre que el autor original y la fuente sean debidamente citados. 


\section{INTRODUÇÃO}

A Enfermagem é uma profissão que atua diretamente no desenvolvimento de ações de promoção da saúde, prevenção de doenças e reabilitação da saúde da população. Devido a este trabalho ser caracterizado por demandas de alta complexidade, tem um alto risco de desenvolver estresse psicológico e físico, independentemente do local de atuação profissional ${ }^{1}$.

Concatenado ao estresse, surgem outros fatores que auxiliam no desgaste físico e mental, como por exemplo, condições de trabalho precárias, altas jornadas e sobrecarga de trabalho, exposição a fatores de riscos, desmotivação profissional, baixa remuneração e dupla jornada de serviços, o que resulta em reflexos negativos na qualidade de vida $(\mathrm{QV})$ desses profissionais ${ }^{2}$.

Nesse contexto, salienta-se que a QV compreende inúmeros fatores, dentre eles destacam-se a saúde física e psicológica, nível de independência, relacionamentos sociais, interações com família, amigos e o próprio meio ambiente ${ }^{3}$.

A literatura aponta a influência negativa na qualidade de vida de trabalhadores da área de saúde oriunda do contato rotineiro com a dor, sofrimento, terminalidade da vida, expectativa do usuário do sistema de saúde e as limitações do sistema assistencial. Como agravante, cita-se o fato de que alguns profissionais possuem mais de um vínculo empregatício, o que resulta em um grande desgaste físico e mental. Ademais, o elevado nível de estresse comum ao setor de trabalho, acarreta em riscos de falhas durante o processo assistencial, o que reflete diretamente na segurança do cuidado prestado ${ }^{4}$.

Tais situações podem se apresentar de maneira significativa entre os profissionais de enfermagem atuantes em setores críticos (Unidades de Terapia Intensiva - UTIs; Centro Cirúrgico- $\mathrm{CC}$; e, Pronto Socorro - PS), pelo fato de lidarem diariamente com paradoxos como a vida e morte.

Em decorrência das inúmeras demandas de alta complexidade, nesses setores pode haver uma exposição maior a riscos ocupacionais referente às atividades desenvolvidas, como os riscos físicos (estrutura, iluminação, temperatura do ambiente), químicos (medicamentos), biológicos (micro-organismos), psicossocial (estresse, fadiga, insatisfação) e o ergonômico (postura incorreta, excesso de peso $)^{5}$.

Os serviços desenvolvidos no CC, UTIs e PS são destinados, de um modo geral, para pacientes críticos e semi-críticos, que necessitam de cuidados especializados em tempo hábil, tomada de decisão correta e utilização de recursos tecnológicos avançados para a monitorização constante dos parâmetros vitais. A rotina de trabalho nesses setores marcada pela alta complexidade expõe a equipe de enfermagem ao estresse prolongado e acarreta repercussões a segurança do paciente e a qualidade do cuidado prestado ${ }^{6,7}$. Entretanto, pouco se tem discutido sobre sua qualidade de vida desses profissionais. Neste sentido, questiona-se: Como se apresenta a qualidade de vida entre profissionais de enfermagem atuantes em setores críticos? Mediante a isso, o presente estudo tem 
como objetivo analisar a qualidade de vida de profissionais de enfermagem atuantes em setores críticos.

\section{MATERIAIS E MÉTODOS}

Estudo transversal de abordagem quantitativa, realizado no período de 09 de outubro á 15 de novembro de 2016, com a equipe de enfermagem (enfermeiros, técnicos de enfermagem e auxiliares) atuantes em setores críticos, de uma instituição hospitalar filantrópica de direitos privados sem fins lucrativos situada na região noroeste do Paraná, Brasil.

Os setores selecionados para a pesquisa foram o CC, as UTIs (adulto e neonatal) e o PS por constituírem setores fechados e os profissionais lidarem diariamente com o limiar de vida ou de morte dos pacientes. Além disso, são locais que possuem expressivo risco de transmissão de infecções, devido à assistência oferecida a pacientes graves, ou com risco de contaminação por conta dos procedimentos cirúrgicos, pacientes com quadros clínicos graves e risco de complicações repentinas.

Para coleta de dados utilizou-se dois instrumentos, sendo o primeiro um questionário semiestruturado elaborado pelos pesquisadores para a caracterização sociodemográfica, que continha questões relacionadas ao sexo, idade, estado civil, escolaridade e formação profissional. O segundo questionário utilizado foi o $W H O Q O L-$ $B R E F$, previamente validado para o Brasil por Fleck, Chachamovich e Trentini em $2006^{8}$.

O WHOQOL-BREF é um questionário autoaplicável que contém 26 questões, divididas em quatro domínios. As respostas são compostas em escala do tipo Likert de cinco pontos, e sua pontuação varia de 38 a 118 pontos. Cumpre mencionar, que os autores não estabeleceram ponto de corte para classificar a QV como excelente boa ou ruim, desta forma, considerouse que quanto maior a pontuação do instrumento melhor a percepção da QV entre os participantes.

A aplicação dos questionários ocorreu após agendamento prévio junto aos enfermeiros atuantes nos diferentes setores, de acordo com a disponibilidade de cada profissional participante, sendo que os pesquisadores aguardavam o término de preenchimento do questionário.

Foram convidados a participar os 88 profissionais atuantes nos setores críticos da instituição investigada, destes 75 aceitaram participar e atenderam aos critérios de inclusão sendo eles: ser enfermeiro, técnico de enfermagem ou auxiliar de enfermagem e atuar no referido setor há pelo menos seis meses. Assim, foram incluídos: 11 do Pronto Socorro; 26 do Centro Cirúrgico; 23 da Unidade de Terapia Intensiva Adulto e; 15 da Unidade de Terapia Intensiva Neonatal (UTINeo). Foram excluídos oito profissionais de atestado médicollicença e, cinco se recusaram a participar.

Os dados foram transcritos em planilhas eletrônicas e analisados por meio de estatística descritiva, com medidas de tendência central e dispersão. Seguindo as recomendações dos autores $^{8}$, as questões três, quatro e 26 foram interpretadas de maneira inversa. Como os dados 
não seguem distribuição normal optou-se por utilizar a mediana para representá-los.

Os preceitos éticos que envolvem pesquisa com seres humanos foram respeitados, e o projeto desta foi aprovado sob parecer de $\mathrm{n}^{\mathrm{o}} 1.760 .486$ do Comitê de Ética em Pesquisa com Seres Humanos da Universidade Estadual de Maringá atendendo a Resolução 466/2012 do Conselho Nacional de Saúde/Ministério da Saúde. Todos os participantes preencheram o Termo de Consentimento Livre e Esclarecido em duas vias, ficando uma via em poder do profissional entrevistado.

\section{RESULTADOS}

Os 75 trabalhadores de enfermagem investigados tinham em média 30 anos. Os demais dados de caracterização estão apresentados na Tabela 1.

Tabela 1. Caracterização dos profissionais de enfermagem atuantes em setores críticos de uma instituição hospitalar do noroeste do Paraná, Brasil, 2016

\begin{tabular}{|c|c|c|c|c|c|c|c|c|c|c|}
\hline \multirow[t]{2}{*}{ Variável } & \multicolumn{2}{|c|}{$\begin{array}{c}\mathrm{CC} \\
(n=26)\end{array}$} & \multicolumn{2}{|c|}{$\begin{array}{l}\text { UTI-A } \\
(n=23)\end{array}$} & \multicolumn{2}{|c|}{$\begin{array}{c}\text { UTI - NEO } \\
(\mathbf{n}=\mathbf{1 5})\end{array}$} & \multicolumn{2}{|c|}{$\begin{array}{c}\text { PS } \\
(n=11)\end{array}$} & \multicolumn{2}{|c|}{$\begin{array}{c}\text { Total } \\
(n=75)\end{array}$} \\
\hline & $\mathbf{n}$ & $(\%)$ & $\mathbf{n}$ & $(\%)$ & $\mathbf{n}$ & $(\%)$ & $\mathbf{n}$ & $(\%)$ & $\mathbf{n}$ & $(\%)$ \\
\hline \multicolumn{11}{|l|}{ Sexo } \\
\hline Feminino & 19 & 73 & 17 & 74 & 15 & 100 & 8 & 73 & 59 & 79 \\
\hline Masculino & 7 & 27 & 6 & 26 & & - & 3 & 27 & 16 & 21 \\
\hline \multicolumn{11}{|l|}{ Estado Civil } \\
\hline Solteiro & 9 & 36 & 9 & 39 & 7 & 47 & 6 & 54 & 31 & 41 \\
\hline Casado/União estável & 15 & 58 & 12 & 52 & 8 & 53 & 5 & 46 & 40 & 53 \\
\hline Divorciado & 2 & 8 & 2 & 9 & & - & & - & 4 & 6 \\
\hline \multicolumn{11}{|l|}{ escolaridade } \\
\hline Ensino Médio e Técnico & 21 & 81 & 16 & 70 & 11 & 73 & 7 & 64 & 55 & 73 \\
\hline Ensino superior incompleto & 2 & 8 & 1 & 4 & - & - & & & 3 & 4 \\
\hline Ensino superior completo & 2 & 8 & 3 & 13 & 1 & 7 & 2 & 18 & 8 & 11 \\
\hline Especialização & 1 & 3 & 3 & 13 & 3 & 20 & 2 & 18 & 9 & 12 \\
\hline \multicolumn{11}{|l|}{ Formação Profissional } \\
\hline Técnico de Enfermagem & 25 & 96 & 19 & 83 & 10 & 67 & 8 & 73 & 62 & 83 \\
\hline Enfermeiro & 1 & 4 & 4 & 17 & 4 & 27 & 3 & 27 & 12 & 16 \\
\hline $\begin{array}{l}\text { Estagiário de Téc. de } \\
\text { Enfermagem }\end{array}$ & & - & & - & 1 & 7 & & & 1 & 1 \\
\hline
\end{tabular}

CC: Centro cirúrgico; UTI-A: Unidade de Terapia Intensiva-Adulto; UTI-NEO: Unidade de Terapia Intensiva-Neonatal; PS: Pronto Socorro. 
Em média os profissionais atuam há seis anos na instituição; com carga horária semanal de 42 horas; levam cerca de 10 minutos de deslocamento até o trabalho (mínimo de cinco minutos, e máximo de 60). Ainda, $45(60 \%)$ profissionais referiram estar satisfeito com seu trabalho; $13(17,3 \%)$ muito satisfeito; $12(16 \%)$ neutro; quatro $(5,3 \%)$ insatisfeitos; e um $(1,3 \%)$ muito insatisfeito.

A Tabela 2, apresenta a análise por domínio geral, entre todos os profissionais atuantes em setores críticos da instituição investigada.

Tabela 2. Análise por domínios do instrumento WHOQOL-Bref entre profissionais de enfermagem atuantes em setores críticos em uma instituição hospitalar do noroeste do Paraná, Brasil, 2016

\begin{tabular}{|l|c|c|c|}
\hline \multicolumn{1}{|c|}{ Domínios } & Mediana & Mínimo & Máximo \\
\hline Físico & 56,48 & 32 & 82 \\
\hline Psicológico & 65,33 & 33 & 83 \\
\hline Relação social & 76,89 & 30,0 & 80 \\
\hline Ambiente & 61,67 & 38,0 & 88 \\
\hline Total & 73,33 & 35 & 97 \\
\hline
\end{tabular}

A Tabela 3 apresenta a análise por domínio do instrumento que avaliou a qualidade de vida dos profissionais, divididos por setores de atuação.

Tabela 3. Análise por domínios e setores de atuação segundo WHOQOL-Bref entre profissionais de enfermagem de uma instituição hospitalar do noroeste do Paraná, Brasil, 2016

\begin{tabular}{|l|c|c|c|c|}
\hline \multicolumn{1}{|c|}{ Domínios } & CC & UTI-Adulto & UTI-Neo & PS \\
\hline Físico & 57,28 & 55,59 & 55,47 & 57,79 \\
\hline Psicológico & 65,22 & 67,57 & 60,83 & 67,04 \\
\hline Relação social & 77,56 & 78,98 & 72,77 & 76,51 \\
\hline Ambiente & 60,81 & 60,05 & 61,45 & 67,32 \\
\hline Total & 77,40 & 72,82 & 64,16 & 77,27 \\
\hline
\end{tabular}

CC: Centro cirúrgico; UTI-A: Unidade de Terapia Intensiva-Adulto; UTI-NEO: Unidade de Terapia Intensiva-Neonatal; PS: Pronto Socorro. 


\section{DISCUSSÃO}

De acordo com a caracterização sociodemográfica deste estudo, houve prevalência de técnicos de enfermagem $(82,7 \%)$, do sexo feminino $(78,7 \%)$, adultos jovens $( \pm 30$ anos), e casados $(53,3 \%)$, conforme observa-se na Tabela 1. Embora os homens estejam gradativamente se inserindo nos campos de trabalho da enfermagem, ainda é comum às mulheres serem maioria na profissão 9 .

A prevalência de técnicos de enfermagem se relaciona ao fato de que no contexto brasileiro a enfermagem exerce um trabalho hierarquizado, em que, atribui-se a concepção e organização do cuidado aos enfermeiros e, a maior demanda assistencial aos técnicos de enfermagem. Entretanto, quando se trata de setores críticos, destaca-se que segundo a lei que rege o exercício profissional da enfermagem no referido país ${ }^{10}$, cabe privativamente aos enfermeiros a assistência à pacientes graves com risco de vida e cuidados de enfermagem de maior complexidade técnica, que exijam conhecimentos de base científica e a capacidade de tomar decisões imediatas. Dessa forma, ressalta-se o fato da alocação de pessoal na instituição permitir maiores proporções de profissionais técnicos de enfermagem em relação à enfermeiros em setores considerados críticos, pode permitir a ocorrência de possíveis desvios de função que podem comprometer o cuidado prestado.

Ainda, 60\% dos participantes referiram estar satisfeitos com seu trabalho, e 17,3\% relataram estar muito satisfeito. Tal dado contradiz um estudo recente no qual teve por objetivo determinar o índice de satisfação no trabalho na equipe de enfermagem na Unidade de Terapia Intensiva Adulto e obteve como resultado insatisfação no trabalho relacionada a falta de reconhecimento da profissão, dificuldade de interação e baixa remuneração ${ }^{11}$. A divergência dos dados encontrados nesta investigação com estudos anteriores pode estar relacionada à proximidade entre a moradia e a instituição (média de 10 minutos de deslocamento), bem como, ao fato de que os profissionais atuam em carga horária de 42 horas semanais, ou seja, menor que investigação anterior ${ }^{6}$. Destaca-se que a redução da carga horária semanal para 40 horas é defendida em artigo recente como forma de melhoria da qualidade de vida entre trabalhadores ${ }^{6}$.

No escore geral, os profissionais totalizaram uma QV que pode ser interpretada como satisfatória $(73,33)$, por finalizarem com valor mais próximo de 118 pontos. Os maiores escores de QV foram encontrados nos domínios Relação Social $(76,89)$ e Psicológico $(65,33)$, o que pode indicar que os profissionais, de modo geral, possuem redes de apoio bem estabelecidas e estabilidade emocional condizente com o exigido pela função desempenhada, refletindo positivamente em sua saúde mental.

O domínio Relação Social diz respeito aos relacionamentos pessoais, sociais e suporte familiar, e a forma em que essas situações podem afetar os trabalhadores psicologicamente. Nesta perspectiva, o apoio social auxilia no enfrentamento das mudanças e ritmos cotidianos da vida, mudanças essas relacionadas com o meio físico e psicológico ${ }^{12}$. 
O trabalho dos profissionais de enfermagem no ambiente hospitalar ocorre em regime de plantões, influencia o cotidiano desses trabalhadores e como consequência sua qualidade de vida. Isto porque, o profissional tende a estar ausente em eventos e confraternizações, bem como, em momentos de fortalecimento de laços familiares, tais como a possibilidade de brincar com o filho ou colocá-lo para dormir ${ }^{13}$.

Dessa forma, destaca-se que ao classificar o domínio Relação Social como satisfatório, os profissionais investigados demonstraram que recebem o apoio e a compreensão necessária para permanência na profissão. Neste sentido, percebe-se que as relações sociais influenciam diretamente na saúde mental dos trabalhadores, servindo de suporte para o enfrentamento dos momentos difíceis da vida, já que o apoio social auxilia na elaboração de estratégias de coping pelos indivíduos.

O domínio Psicológico diz respeito à ansiedade e ao estresse, que muitos profissionais são submetidos diariamente durante os turnos de trabalho ${ }^{14}$, seja por situações cotidianas ou por desordens dentro e/ou fora do ambiente de trabalho. Nesse aspecto, considera-se que os desequilíbrios de ordem psicológica podem impactar negativamente no desempenho das funções laborais de profissionais da enfermagem já que suas atividades demandam estabilidade emocional para lidar com a subjetividade humana.

Ademais, a enfermagem em seu processo de trabalho é caracterizada como uma profissão com probabilidade de sofrer com o impacto do estresse, devido aos cuidados prestados aos indivíduos em fase terminal ou com complicação da patologia, por situações repentinas e imprevisíveis. Essa imprevisibilidade dos desfechos, bem como conflitos pessoais e profissionais gerados pelo confronto entre suas crenças, valores e questões éticas suscitadas pelo cotidiano de trabalho podem afetar substancialmente a dimensão emocional do profissional $^{13}$. Desta forma se favorece o desenvolvimento do estresse ${ }^{14}$, e da ansiedade, visto a necessidade de que este profissional esteja preparado física e emocionalmente para tomadas de decisões clínicas.

Embora a presente investigação levante evidências de uma avaliação positiva em relação ao domínio Psicológico entre os profissionais de enfermagem, o domínio Ambiente que diz respeito às condições de trabalho, obteve pouco mais da metade da pontuação possível no domínio $(61,67)$. Nessa perspectiva, alerta-se para a maior proporção de profissionais técnicos de enfermagem em um setor considerado crítico e que se destina à assistência a doentes graves e cuidados complexos, atividade privativa do enfermeiro ${ }^{10}$, o que pode ser gerador de sobrecarga de trabalho para este profissional.

O domínio Físico apresentou o menor escore entre os Profissionais $(56,48)$. Este se relaciona ao sono/repouso e a capacidade dos profissionais de desenvolver seu trabalho, nesse contexto o exercício do trabalho em turnos ou plantões é um fator gerador de estresse, e pode influenciar na saúde e na qualidade de vida dos profissionais ${ }^{13}$. Estudo que foi realizado com profissionais de 
saúde obteve que trabalhadores fisicamente ativos obtiveram melhores avaliações relacionadas a sua capacidade funcional, vitalidade e saúde mental, em relação aos profissionais inativos ${ }^{1}$, o que pode ser utilizado por gestores como forma de enfrentamento e melhoria da qualidade de vida entre os profissionais.

Investigação realizada em um hospital de Minas Gerais que teve o objetivo de identificar os sintomas referentes ao estado geral de saúde associado ao trabalho em turnos de enfermagem, e relacioná-los com a qualidade do sono, apontou que a pior qualidade de sono noturno era mais presente em trabalhadores do turno diurno que apresentaram sintomas como má digestão e irritabilidade. Ainda demonstrou alterações na saúde física e psicológica dos profissionais de enfermagem, após atuar em turnos, seja diurno ou noturno ${ }^{16}$. Tal fato remonta a necessidade de investimento por parte da instituição para mitigar o impacto que o trabalho nos serviços hospitalares gera na saúde e qualidade de vida dos profissionais.

Ressalta-se, nesse aspecto, a importância dos períodos de descanso e sono adequados a fim de minimizar os efeitos do estresse, próprios da atuação profissional, sobre o corpo e a mente do trabalhador. Ter boa $\mathrm{QV}$ relacionada à saúde física pode influenciar positivamente em variadas dimensões da vida do indivíduo e favorecer o desempenho nas atividades laborais.

Com relação à QV dos participantes situados em diferentes setores observa-se que os menores escores foram encontrados nos domínios Físico e
Ambiente. O baixo resultado no Domínio Físico destoa do resultado obtido em estudo recente, que teve por objetivo avaliar a QV de profissionais de enfermagem da UTI de um hospital filantrópico no interior do Paraná e obteve que o domínio Físico é o melhor percebido pelos enfermeiros da unidade $^{17}$.

No domínio Físico a UTI-A $(55,59)$ e UTINeo $(55,47)$ apresentaram os menores escores, por vezes justificado pela alta carga de estresse que permeia este ambiente. Isso porque, ao contrário de setores como o PS e o CC em que os pacientes são acompanhados por um período curto de tempo, nas UTIs o tempo de internamento é maior, o que resulta na interação social prolongada com os pacientes e seus familiares, podendo gerar vínculo. Desta forma, as dimensões físicas e psicológicas fragilizadas influenciam negativamente no trabalho da equipe multiprofissional na terapia intensiva e revelam o conflito entre o dever da manutenção da vida e o processo de finitude ${ }^{20}$.

No Domínio Meio Ambiente os menores escores foram nas UTIs e CC. Ao confrontar com a literatura, pode-se identificar que os fatores influenciadores são: as condições de trabalho muitas vezes precárias, a disponibilidade de recursos humanos e de materiais e na estruturação, a organização do processo de trabalho como distribuição de funções e a oportunidade de adquirir novos conhecimentos ${ }^{18}$. Em especial no $\mathrm{CC}$, estudo recente que investigou o clima organizacional no referido setor, obteve que a insatisfação dos trabalhadores está associada, entre outros fatores, às condições de trabalho e o 
número de materiais hospitalares e equipamentos insuficientes para a demanda de cuidado ${ }^{19}$, fatores estes ligados ao ambiente e que influenciam na qualidade de vida dos trabalhadores.

Evidencia-se, assim, que as condições oferecidas pelo ambiente de trabalho podem afetar diretamente a prática do profissional de enfermagem. $O$ ambiente em estudo possivelmente tem desencadeado desconfortos à medida que os trabalhadores manifestaram sua insatisfação com o espaço no qual atuam.

Os achados que representam os menores escores, portanto, sugerem a necessidade de olhar atentamente para os prejuízos físicos e emocionais que o profissional pode sofrer em decorrência do descanso comprometido e da sobrecarga gerada por sua prática cotidiana. Além disso, deve-se primar por ambientes que ofereçam as condições apropriadas para o exercício do cuidado, o que promoverá o bom desempenho do profissional e satisfação em realizar seu trabalho com a qualidade almejada.

Como principais limitações do estudo, citase o fato de ter sido realizado em uma única instituição com uma realidade específica e a não realização de cálculo amostral. Entretanto, seus resultados podem servir de auxílio para melhoria da qualidade de vida no trabalho daqueles que vivenciam realidade semelhante. Futuras pesquisas precisam ser realizadas com a proposta de compreender, na percepção do trabalhador, os fatores que influenciam em sua $\mathrm{QV}$, além de analisar e implementar açõeslestratégias que visam melhorar a qualidade de vida no trabalho desses profissionais.

\section{CONCLUSÕES}

$\mathrm{O}$ Escore total de QV dos profissionais de enfermagem atuantes em setores críticos foi relativamente elevado. Entretanto, observa-se que o domínio Físico finalizou com uma avaliação insatisfatória em todos os setores, o que sugere a necessidade de que os gestores atentem para essa demanda, oferecendo as condições necessárias para minimizar tais prejuízos.

O domínio Relações Sociais finalizou com as melhores pontuações, o que indica que os profissionais possuem uma rede de apoio bem estabelecida.

Os resultados obtidos no presente estudo, portanto, explicitam a proporção que o comprometimento da QV, ainda que em alguns aspectos, toma no contexto de sua prática de trabalho. Variadas dimensões de sua vida são afetadas o que acarreta prejuízos diretos à saúde física e mental do trabalhador bem como à qualidade da assistência prestada. Nesse sentido espera-se que essas evidências possam motivar a promoção da QV desses profissionais, para assim exercerem seu ofício com saúde, satisfação e prazer.

Conflito de interesses: Os autores declaram que não há conflito de interesses. 


\section{REFERÊNCIAS}

1. Acioli NA, Araújo R, Pitangui A, Menezes L, França E, Costa E, et al. Qualidade de vida e nível de atividade física de profissionais de saúde de unidades de terapia intensiva. Rev. bras. ativ. fis. saúde. 2013; 18(6): 711-9. https://doi.org/10.12820/rbafs.v.18n6p711

2. Maria AL. Síndrome de Burnout em diferentes áreas profissionais e seus efeitos. Acta Brasileira do Movimento Humano. 2016; 6(3): 1-12.

3. The Whoqol Group. The development of the World Health Organization quality of life assessment instrument (the whoqol). In: Orley J, Kuyken W, editors. Quality of life assessment: international perspectives. Heidelberg: Springer Verlag; 1994. p. 41-60.

https://doi.org/10.1007/978-3-642-79123-9

4. Rodrigues CCFM, Santos VEP, Sousa P. Segurança do paciente e enfermagem: interface com estresse e Síndrome de Burnout. Rev. Bras. Enferm. 2017; 79(5): 1141-7. http://dx.doi.org/10.1590/0034-7167-2016-0194

5. Nazario EG, Camponogara S, Dias GL. Riscos ocupacionais e adesão a precauções-padrão no trabalho de enfermagem em terapia intensiva: percepções de trabalhadores. Rev. bras. saúde ocup. 2017; 42: e7. http://dx.doi.org/10.1590/2317-6369000009216

6. Souza JD, Júnior JMP, Miranda FAN. Stresse em serviço de urgência e os desafios para enfermeiros brasileiros e portugueses. Rev. Enf. Ref. 2017; serIV (12): 107-16. http://dx.doi.org/10.12707/RIV16064

7. Azevedo BS, Nery AA, Cardoso JP. Estresse ocupacional e insatisfação com a qualidade de vida no trabalho da enfermagem. Texto \& contexto enferm. 2017; 26(1): 1-11. http://dx.doi.org/10.1590/0104-07072017003940015

8. Fleck MPA, Chachamovich E, Trentini C. Development and validation of the Portuguese version of the whoqolold module. Rev Saude Publica. 2006; 40(5): 785-91. http://dx.doi.org/10.1590/S0034-89102006000600007

9. Pimenta AM, Assunção AA. Estresse no trabalho e hipertensão arterial em profissionais de enfermagem da rede municipal de saúde de Belo Horizonte, Minas Gerais, Brasil. Rev. bras. saúde Ocup. 2016; 41: e6. http://dx.doi.org/10.1590/2317-6369000113515

10. Conselho Federal de Enfermagem. Lei n. 7.498, de 25 de junho de 1986. Dispõe sobre a regulamentação do exercício da enfermagem e dá outras providências. Diário Oficial da República Federativa do Brasil, Brasília (DF); 1986; Seção 1.

11. Versa GL, Matsuda LM. Satisfação profissional da enfermagem intensivista. Rev. enferm. UERJ. 2014; 22(3): 409-15.

12. Dimenstein M, Siqueira K, Macedo JP, Leite J, Dantas C. Determinação social de saúde mental: contribuições a psicologia no cuidado territorial. Arq. bras. psicol. 2017; 69(2): 72-87.
13. Santana RS, Brito BA, Ferreira JL, Silva AF, Cunha MB, Viana LV. Influência do trabalho noturno na qualidade de vida da equipe de enfermagem da UTI. Rev. Interd. 2015; 8(2): 25-34.

14. Oliveira LP, Araújo GF. Características da síndrome de burnout em enfermeiros da emergência de um hospital público. Revista Enfermagem Contemporânea. 2016; 5(1): 34-42.

http://dx.doi.org/10.17267/2317-3378rec.v5i1.834

15. Vega MEP, Cibanal LJ. Impacto psicosocial en enfermeras que brindan cuidados en fase terminal. Rev Cuid. 2016; 7(1): 1210-8.

http://dx.doi.org/10.15649/cuidarte.v7i1.295

16. Mendes SS, Martino MMF. Trabalho em turnos: estado geral de saúde relacionado ao sono em trabalhadores em enfermagem. Rev. Esc. Enferm. USP. 2012; 46(6): 1471-6. https://doi.org/10.1590/S0080-62342012000600026

17. Volpato YGF, Martins MR. Avaliação da qualidade de vida da equipe de enfermagem da UTI geral de um hospital filantrópico no interior do Paraná. Revista UNINGÁ. 2013; 36: $81-8$.

18. Mascarenhas CHM, Prado FO, Fernandes MH, Boeri EN, Sena ELS. Qualidade de vida em trabalhadores da área de saúde: uma revisão sistemática; Espaç. Saúde (Online). 2013; 14(1/2): 1-10.

19. Chaves JA, Guimarães MGV. Análise do clima organizacional em centro cirúrgico de um Hospital Universitário da cidade de Manaus. Revista FAROL. 2016; 1(1): 206-19.

20. Hercos TM, Vieira FS, Oliveira MS, Buetto LS, Shimura CMN, Sonobe HM. O Trabalho dos Profissionais de Enfermagem em Unidades de Terapia Intensiva na Assistência ao Paciente Oncológico. Rev. Bras. Cancerol. (Online). 2014; 60(1): 51-8. 